Relations industrielles

Industrial Relations

\title{
La lutte contre la discrimination dans le travail, Bureau International du Travail, Cours d'éducation ouvrière, Genève, 1968, 218 pp.
}

\section{Bernard Solasse}

Volume 23, numéro 2, 1968

URI : https://id.erudit.org/iderudit/027903ar

DOI : https://doi.org/10.7202/027903ar

Aller au sommaire du numéro

Éditeur(s)

Département des relations industrielles de l'Université Laval

ISSN

0034-379X (imprimé)

1703-8138 (numérique)

Découvrir la revue

Citer ce compte rendu

Solasse, B. (1968). Compte rendu de [La lutte contre la discrimination dans le travail, Bureau International du Travail, Cours d'éducation ouvrière, Genève, 1968, 218 pp.] Relations industrielles / Industrial Relations, 23(2), 368-368.

https://doi.org/10.7202/027903ar

Tous droits réservés (C Département des relations industrielles de l'Université Laval, 1968
Ce document est protégé par la loi sur le droit d'auteur. L’utilisation des services d’Érudit (y compris la reproduction) est assujettie à sa politique d'utilisation que vous pouvez consulter en ligne.

https://apropos.erudit.org/fr/usagers/politique-dutilisation/ 


\section{RECENSIONS}

La lutte contre la discrimination dans le travail, Bureau International du Travail, Cours d'éducation ouvrière, Genève, 1968, $218 \mathrm{pp}$

Ce cours d'éducation ouvrière complète une série de publications antérieures dont il est bon de rappeler les titres car elles constituent autant d'ouvrages de référence utiles surtout aux étudiants: La coopérotion (1956) - La sécurité sociale (1958) La liberté syndicale (1959) - Les négociations collectives (1960) - La prévention des accidents (1961) — Les salaires (1964) - Le monde du travail face à l'âge nouveau (1965).

Ce dernier cours est précieux à plusieurs égards

11 contient une définition de la nature et une description des forces les plus fréquentes de discrimination dans le travail (Leçons 1 ef 4)

Certaines formes de discrimination dans le travail sont l'objet d'études particulières; telles les discriminations fondées sur la race et la couleur, le sexe, la religion, les opinions politiques, les origines sociales et cette forme de discrimination raciale particulièrement odieuse qu'est I' « apartheid ».

Deux leçons (les leçons 2 et 3 ) sont consacrées à l'exposé des positions de l'Organisation internationale du travail sur ces questions. Une troisième leçon (la leçon 12) résume les actions entreprises par l'Organisation internationale du travail et par d'autres organisations internationales afin de mettre fin à ces formes de discrimination. Une leçon est consacrée à l'action des autorités nationales contre la discrimination. Une distinction est établie entre les pays qui ont ratifié les conventions de l'Organisation internationale du travail sur ces questions et ceux qui ne les ont pas ratifiées. A ce niveau, le cours ne suggère que des orientations générales puisque l'Organisation internationale du travail ne peut s'ingérer dans les affaires intérieures d'un Etat.

Les deux dernières leçons s'adressent directement oux organisations de trovailleurs et d'employeurs et contiennent un ensemble de suggestions pratiques concernant les mesures et les procédures qui devraient permet- tre de lutter efficacement contre les différentes formes de discrimination dans le travail.

On notera en annexe, la pubication de larges extraits des conventions et des recommandations adoptées par l'Organisation internotionale du trovail. Leur intérêt documentaire est évident et s'ajoute ou foit que ces textes peuvent nourrir l'ougmentation d'organisations syndicales appelées à lutter contre la discrimination dons le travail.

La bibliographie reste limitée oux publicotions officielles du Bureau internotional du travail

\section{Bernard SOLASSE}

Scientists in Organizations - Productive Climates for Research and Development, par Donald C. Pelz et Frank M. Andrews, John Wiley and Sons, Inc., 1956, xii plus 317 pp

Ce livre traite du rendement des travailleurs scientifiques, ou plutôt des conditions de travail qui permettent de maintenir et de prolonger l'efficacité des hommes de science. Les travailleurs scientifiques ont été classifiés selon qu'ils oeuvrent dans le domaine de la recherche fondamentale ou celui de la recherche appliquée ou encore selon le degré de l'éducation formelle qu'ils ont reçue.

D'intéressantes conclusions ont été obtenues: par exemple en ce qui concerne l'importance de varier l'expérience des scientifiques dès les premières étapes de leur carrière. II ne s'agit pas d'établir une politique de formation de la main-d'oeuvre savante qui permettrait aux chercheurs de participer à des trovoux nombreux et superficiels dans divers domaines: car il demeure toujours vrai qu'une spécialisation poussée est la pierre d'angle de toute formation scientifique vraie. Néonmoins un élargissement des connaissances et de l'expérience acquise par des recherches effectuées en dehors du domaine strict de la spécialisation, compte parmi les éléments les plus valables de la formation de base. La porticipation d̀ l'odministration et au processus de décision est également susceptible d'aider à maintenir l'intérêt et l'efficacité des chercheurs. Cependant 